\title{
DOS NUEVAS INSCRIPCIONES Y ALGUNAS CORRECCIONES A LA EPIGRAFÍA ROMANA DE LA PROVINCIA DE ALICANTE
}

\author{
MANUEL ABILIO RABANAL ALONSO \\ JUAN MANUEL ABASCAL PALAZÓN \\ Universidad de Alicante
}

\begin{abstract}
Se completan algunos datos de inscripciones publicadas en el número anterior, al tiempo que se estudian nuevas inscripciones inéditas.
\end{abstract}

In this paper we make new reflexions about inscriptions published in the former volume, and other unpublished inscriptions are added.

Tras la publicación de nuestro primer trabajo de recopilación de las inscripciones romanas de la provincia de Alicante (RABANAL, M. A., y ABASCAL, J. M., Lucentum IV, 1985, 191-244), son varios los epígrafes que han seguido apareciendo en diferentes puntos de la misma zona, en parte debido a las tareas de excavación que se desarrollan en yacimientos arqueológicos ya conocidos, y en parte merced a hallazgos casuales. Presentamos en este trabajo dos nuevas inscripciones funerarias que tienen, en sí mismas, un significado propio. La n. ${ }^{\circ} 1$ procede de las inmediaciones del Tossal de Manises, de donde ya dimos a conocer un nutrido lote de piezas (RABANAL-ABASCAL, 1985, n. ${ }^{\circ} 1-14$ ), y viene a completar, con el testimonio de un emigrante, ese carácter cosmopolita, y de lugar de integración entre lo indígena y lo romano, que debía tener esta ciu- dad del Tossal, abierta a los influjos del mar. La segunda inscripción procede de la localidad de Tárbena, en una comarca escasa en hallazgos romanos, quizá debido a su difícil orografía para cultivos de extensión que excedan la mera economía familiar, y sirve para ensanchar nuestro campo de visión para una época, la romana, que en la provincia de Alicante deja de ceñirse al litoral para abrir las posibilidades de estudio a las comarcas montañosas del interior.

Queremos expresar aquí nuestro agradecimiento a don Enrique Llobregat y a don Rafael Azuar, Director y Conservador del Museo Arqueológico Provincial de Alicante, que nos dieron las máximas facilidades para la publicación de la inscripción del Tossal, así como a don Francisco Soliveres, vecino 
de Tárbena, por su amabilidad al permitirnos estudiar la inscripción conservada en su propiedad.

Núm. 1.-Tossal de Manises (Alicante). Ara funeraria de caliza, fragmentada en su parte derecha, con parte del texto perdido; fue encontrada en la cimentación interior de un edificio en el paraje de «Torre Santiago», cerca del Tossal de Manises, a finales de 1986, y trasladada al Museo Arqueológico Provincial de Alicante, en donde se conserva. Sus dimensiones son $69 \mathrm{cms}$. de altura, $40 \mathrm{cms}$. de anchura y $34 \mathrm{cms}$. de grosor; el campo epigráfico conservado mide $21 \mathrm{cms}$. de alto y $28 \mathrm{cms}$. de ancho. Altura de las letras: 5, 4, 3'5, 3' $5 \mathrm{cms}$. Interpunciones puntuales.

Bibliografía: Inédita.

\section{P(ublius) Fulvius \\ Asclas P[om-] \\ peian(us), ann(orum) \\ $X X X I I, h$ (ic) s(itus) e(st)}

La onomástica del difunto no es habitual en la zona, siendo la primera vez que este nomen y este cognomen aparecen en la epigrafía alicantina. Especial interés reviste la mención del origo, Pompeianus, del que no conocemos otros testimonios en la Península. El cognomen Asclas se ajusta bastante bien al origo citado, pues los testimonios similares proceden casi todos del área itálica, y especialmente de su zona central. La única mención que conocemos en nominativo, Abucius Asclas, procede de Roma (ILS, 1911), aunque quizá se puedan desarrollar en la forma Ascla(s) otros testimonios itáli$\cos$ (CIL XI 7025, 7217), si bien el genitivo Asclae (CIL V 3257, Verona = ILS 3610: Blandus, C.Afini ser.) parece remitir ya a un nominativo $A$ scla, para el que hay otros ejemplos (CIL VIII 2554; CIL X $1985,2107,2162$ y 2326). El estilo del monumento, la fórmula final y la mención del origo permiten fechar la inscripción a mediados del siglo I d. C. (fig. 2).

Núm. 2.-Tárbena. Pequeño bloque funerario en piedra caliza conservado en la partida de Picassaries, en Tárbena, propiedad de don Francisco Soliveres. Se ignora la procedencia exacta de la pieza, pues tradicionalmente se ha conservado en este paraje, sin que su elevado peso y la particular orografía del territorio sugiera un traslado de la misma desde un lugar alejado. Sus dimensiones son $45 \mathrm{cms}$.

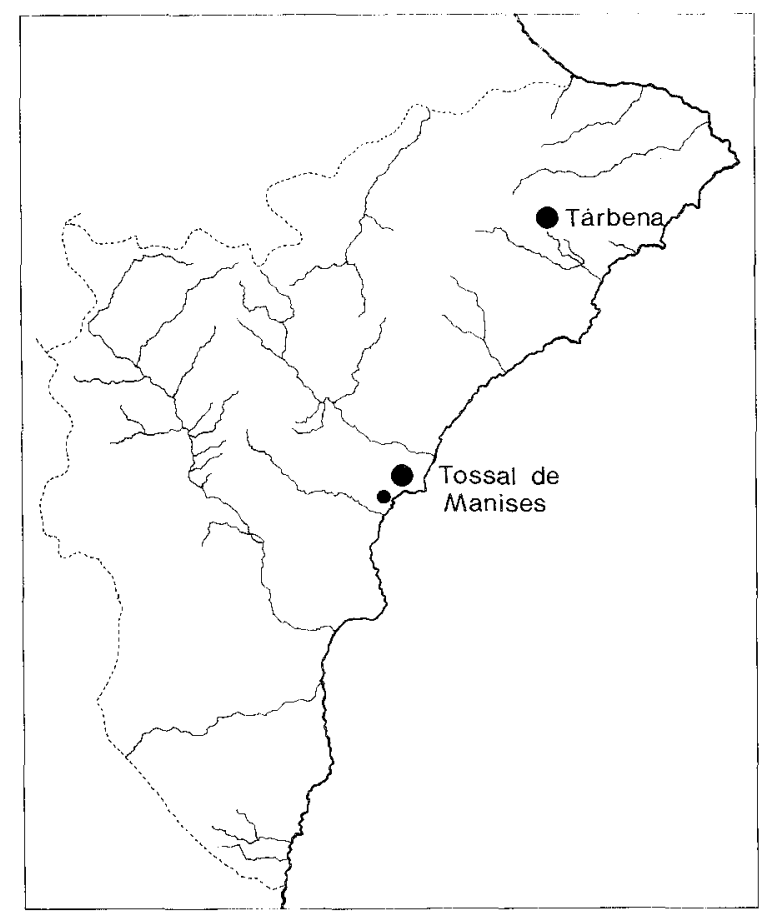

Fig. 1.-Lugares de hallazgo de las nuevas inscripciones. Con un punto de menor tamaño se indica la capital de la provincia para facilitar la localización.

de altura, $32 \mathrm{cms}$. de anchura y $23 \mathrm{cms}$. de grosor. Medidas del campo epigráfico: $22 \mathrm{cms}$. de altura y $17 \mathrm{cms}$. de anchura. Altura de las letras: 4'5, 5 y 3' 5 cms. El texto está enmarcado por una incisión vertical en el lado derecho y presenta interpunciones puntuales en la segunda línea.

Bibliografía: Inédita.

\section{[L]usius L(usii) v(erna) h(ic) s(itus) e(st) an(norum) XXIII!}

La onomástica del difunto no se repite en la epigrafía alicantina, siendo asimismo destacable la infrecuente alteración del orden de la fórmula final y la indicación de edad. El estilo y las características del formulario sugieren una cronología de mediados del siglo I d. C. (fig. 3).

Núm. 3.-Según EE IX, 354a (=ENGEL, Revue arch., ser. III, vol. 29, 1896, 204, nota 3), en las cercanías de Alicante se habría encontrado 


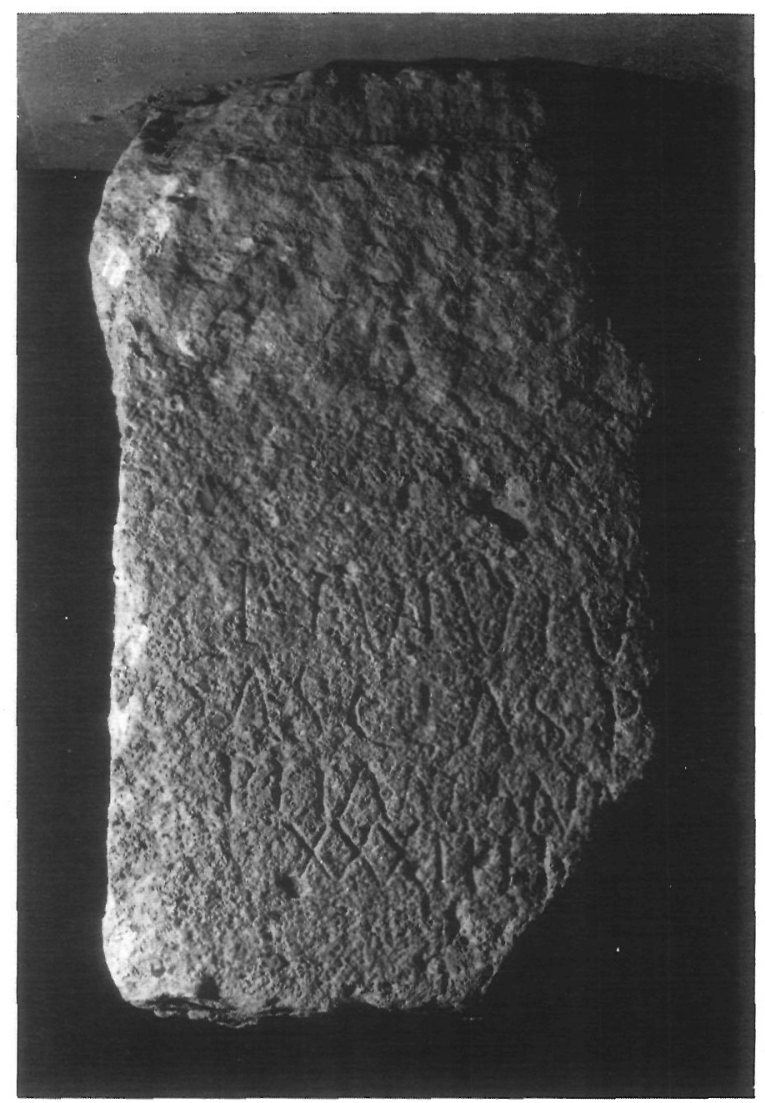

Fig. 2.-Inscripción n. ${ }^{\circ}$ 1. Tossal de Manises.

una inscripción de L. Valerius Nepos, cuyas características y paradero desconocemos.

Núm. 4.-Rabanal-Abascal 1985, 193, n. ${ }^{\circ} 1$, fig. 2: Añádase a la bibliografía EE IX, p. 134, con referencias anteriores, y Memorias de la Sociedad Arqueológica Valenciana 1872(1873), 25, fig. II, 1.

Núm. 5.-Rabanal-Abascal 1985, 193-194, n. ${ }^{\circ}$ 2, fig. 3: Añádase a la bibliografía J. Belda, Museo Arqueológico Provincial de Alicante, en MMAP 6, 1945, 157-165, pp. 164-165, lám. LXXIV, 4.

Núm. 6.-Rabanal-Abascal, 1985, 195, n. ${ }^{\circ} 6$, fig. 6: Encontrada en 1943. Añádase a la bibliografía, J. Belda, Museo Arqueológico Provincial de Alicante, en MMAP 5, 1944, 159-163, p. 163, fig. 16, lám. XLIV, 2.

Núm. 7.-Rabanal-Abascal 1985, 196, n. ${ }^{\circ} 7$, fig. 7: Añádase a la bibliografía J. Belda, Museo Arqueológico Provincial de Alicante, en MMAP 6, 1945, 157-165, p. 165, fig. 23, lám. LXXIV, 5.

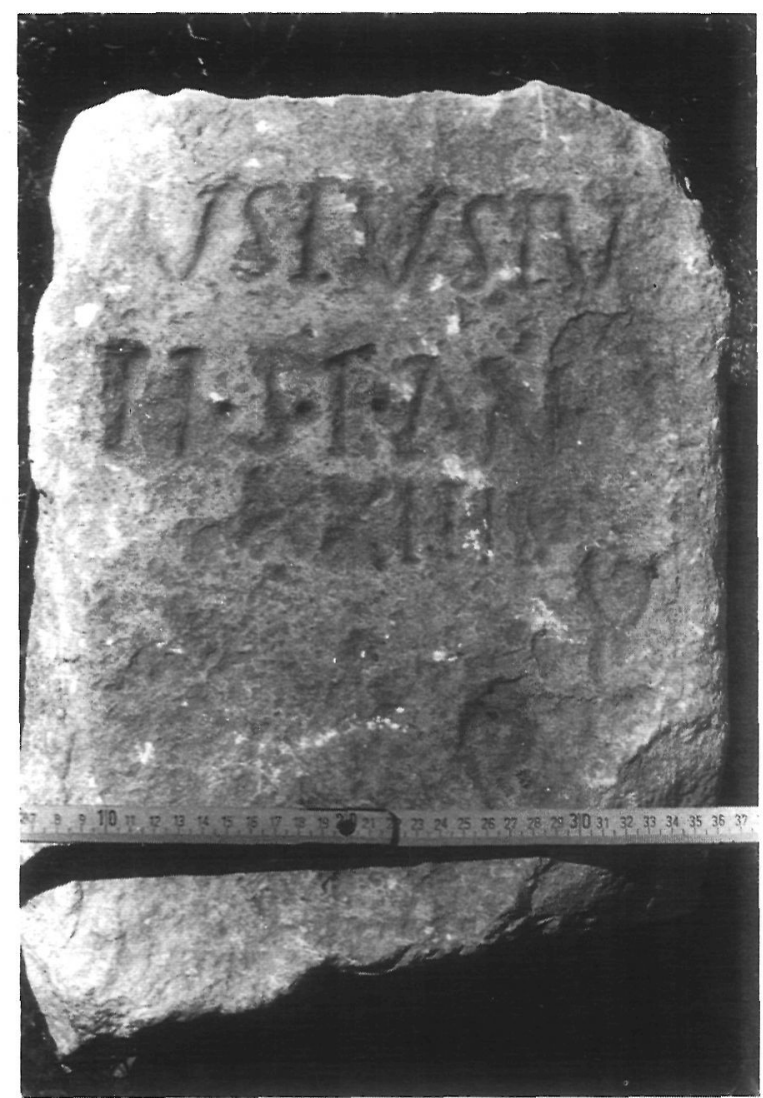

Fig. 3.--Inscripción n. ํ 2. Tárbena.

Núm. 8.-Rabanal-Abascal 1985, 197, n. ${ }^{\circ}$ 8: Añádase a la bibliografía EE VIII, p. 444, CIL I ${ }^{2}$ 2275 (=1482), p. 1106; Degrassi, ILLRP II 622.

Núm. 9.-Rabanal-Abascal 1985, 197, n. ${ }^{\circ} 10$ : Añádase a la bibliografía EE IX, p. 134; ILS 5256a.

Núm. 10.-Rabanal-Abascal 1985, 199, n. ${ }^{\circ} 15$ : Añádase F. Fita, BRAH 12, 1888, 360.

Núm. 11.-Rabanal-Abascal 1985, 205, n。 26: Trasladada a Málaga según EE VIII, p. 444. M. Berlanga, Catálogo del Museo Loringiano (Málaga 1903), 51-52.

Núm. 12.-Rabanal-Abascal 1985, 206-207, n. ${ }^{\circ} 28$, fig. 18: Añádase a la bibliografía, Memorias de la Sociedad Arqueológica Valenciana 1872 (1873), 24, n. ${ }^{\circ} 5=\mathrm{M} .{ }^{a}$ V. Goberna, La sociedad arqueológica valenciana, en APL 16, 1981, 575-608, p. $602, n .^{\circ} 22$. 
Núm. 13.-Rabanal-Abascal 1985, 207-208, n. ${ }^{\circ} 29$, fig. 19: Se observará que la foto ha sido equivocadamente retocada, y que su lectura no concuerda con la transcripción, que sí es correcta.

Núm. 14.-Rabanal-Abascal 1985, 208-209, n. ${ }^{\circ}$ 31, fig. 20: El retoque de la fotografía no es correcto. Léase sólo la transcripción. Sobre la pieza, véase ahora J. J. Ferrer Maestro, Boletín de la Soc. Castellonense de Cultura 61.3, 1985, 367-369.

Núm. 15.-Rabanal-Abascal 1985, 212, n. ${ }^{\circ} 37$, fig. 26: El retoque de la fotografía no es correcto.

Núm. 16.-Rabanal-Abascal 1985, 212-213, N. ${ }^{\circ}$ 38: Hallada recientemente en un domicilio particular de Denia por J. Gisbert. Lo encontrado parece corresponder a la parte izquierda de la pieza, de la que teníamos ya noticias gracias a Chabás. Se trata de una placa de mármol cuyas dimensiones son $27 \times 23 \mathrm{cms}$., no pudiéndose determinar su grosor por estar puesta en el suelo. El texto está mejor conservado de lo que cabía esperar por las noticias antiguas. Vid. ahora fig. 4. Sobre la placa puede leerse:

Aufidia [...]
Saturi [...]
Aufidia [...]
sorores [...]

Núm. 17.-Rabanal-Abascal 1985, 214-215, n. ${ }^{\circ}$ 47, fig. 27: Añádase a la bibligrafía, Memorias de la Sociedad Arqueológica Valenciana 1874-76

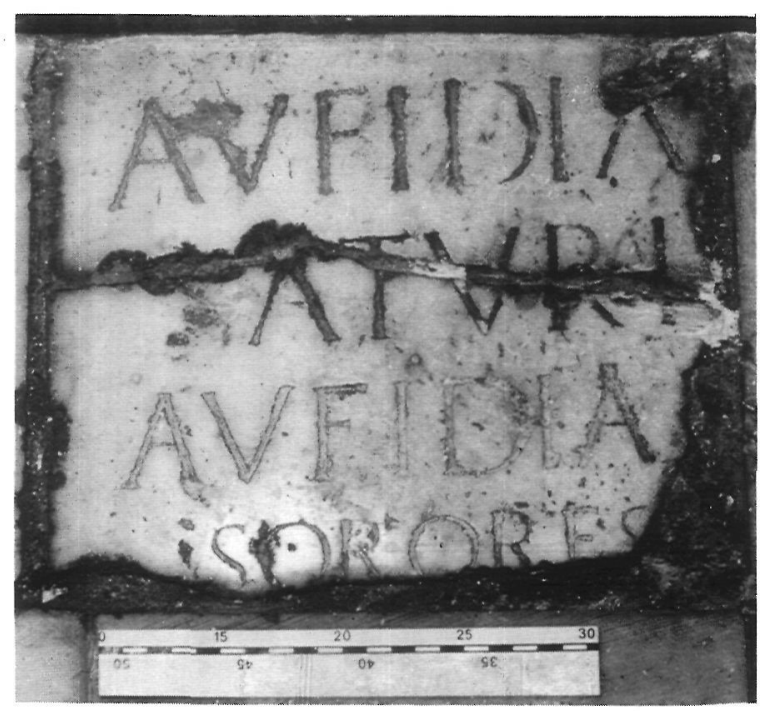

Fig. 4.-Inscripción n. ${ }^{\circ} 16\left(1985\right.$, n. $\left.^{\circ} 38\right)$.
(1877), 34, nota 2, lám. 3, fig. 3 = M. ${ }^{a}$ V. Goberna, APL 16, 1981, 575-608, p. 602, n. ${ }^{\circ} 23$.

Núm. 18.-Rabanal-Abascal 1985, 215-216, n. ${ }^{\circ} 48$, fig. 28: procede del «Huerto de Morand». Añádase a la bibliografía Memorias de la Sociedad Arqueológica Valenciana 1878 (1879), 27-32=M. ${ }^{2}$ V. Goberna, APL 16, 1981, 575-608, p. 602, n. ${ }^{\circ} 24$.

Núm. 19.-Rabanal-Abascal 1985, 216, n. ${ }^{\circ} 49$ : Sobre la pieza véase ahora J. J. Ferrer Maestro, Boletín de la Sociedad Castellonense de Cultura $61.3,1985,367-369$.

Núm. 20.-Rabanal-Abascal 1985, 221, n. ${ }^{\circ} 58$, fig. 34: El retoque de la fotografía no es correcto. Léase sólo la transcripción.

Núm. 21.-Rabanal-Abascal 1985, 223, n. ${ }^{\circ} 62$, fig. 38: En la bibliografía léase EE IX, 349, y añádase HAE 263.

Sobre el topónimo del lugar del hallazgo léase El Alcalet, no Alcaldet. El texto de los dos fragmentos fue publicado de forma continua, cuando no son coincidentes. Pertenecen al primer fragmento las líneas 1 a 8 , mientras que el resto pertenece al segundo.

En la bibliografía deben añadirse, a propósito de la Tabula Hebana, los fundamentales trabajos de J. González y F. Fernández (Tabula Siarensis, en Iura XXXII, 1981, 1-36, lám.) y J. González (Tabula Siarensis: Fortunales Siarenses y municipia civium romanorum, en ZPE 55, 1984, 55-100). En la cita de J. González, Iura 31, 1980, léase pp. 135-137.

Núm. 22.-Rabanal-Abascal 1985, 224-225, n. ${ }^{\circ}$ 64, fig. 40; Añádase a la bibliografía ILS 893.

Núm. 23.-Rabanal-Abascal 1985, 225, n. ${ }^{\circ} 65$, fig. 41: Añádase a la bibliografỉa A. D’Ors, Emerita 28, 1960, 327 ss. = HAE 1971.

Núm. 24.-Rabanal-Abascal 1985, 225, n. ${ }^{\circ} 66$, fig. 42: Añádase a la bibliografía A. D'Ors, Emerita 28, 1960, 327 ss. = HAE 1969.

Núm. 25.-Rabanal-Abascal 1985, 226, n. ${ }^{\circ} 70$, fig. 43: Añádase a la bibliografía EE IX, 350 .

Núm. 26.-Rabanal-Abascal 1985, 228, n. ${ }^{\circ} 74$, fig. 47: Añádase a la bibliografía HAE 1968.

Núm. 27.-Rabanal-Abascal 1985, 229, n. ${ }^{\circ} 75$, fig. 48: La fotografía está invertida. 


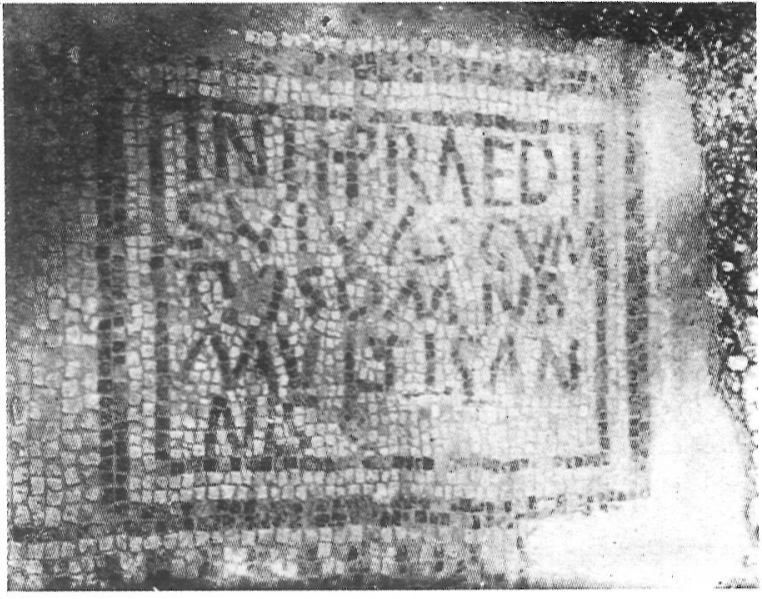

Fig. 5.-Mosaico con inscripción de El Alcalet (Alcudia de Elche). Según Ramos Folqués 1953, fig. 19.

Núm. 28.-Rabanal-Abascal 1985, 229, n. ${ }^{\circ} 76$, fig. 49: Se trata de la misma pieza que la inventariada bajo nuestro número $69(1985,226)$, que dábamos por desaparecida, y que fue publicada por R. Ramos 1975 , n. ${ }^{\circ}$ 6. Añádase a la bibliografía A. D'Ors, Emerita 28, 1960, 327 ss. = HAE 1970.

Núm. 29.-Rabanal-Abascal 1985, 229, n. ${ }^{\circ} 77$, fig. 50: Añádase a la bibliografía A. D'Ors, Emerita 28, 1960, 327 ss. = HAE 1973.

Núm. 30.-Rabanal-Abascal 1985, 231, n. ${ }^{\circ} 83$ : Añádase a la bibliografía $\mathrm{EE} I \mathrm{IX}, 351$, con bibliografía anterior, HAE 4-5, 1953-54, pág. 4 y M. Palomar, De epigrafía española romano-cristiana y visigoda, Zephyrus 2, 1951, 21 ss., n. ${ }^{\circ} 11$. = HAE 381.

En nuestro anterior trabajo, y por haber sido destruido, incluimos esta inscripción tomando su texto de referencias bibliográficas. Una posterior revisión bibliográfica nos ha permitido conocer una fotografía de la misma antes de su destrucción, sin que sea preciso modificar su lectura. El texto figura en un mosaico procedente de la hacienda El Alcalet, y está escrito en 5 líneas de teselas oscuras sobre fondo blanco, enmarcadas en un doble rectángulo que se encuentra adosado a una de las esquinas del mosaico en cuestión. Fue recogido por Ibarra antes de su destrucción y publicado por Ramos Folqués (1953, fig. 19), con dibujo del propio Ibarra. El tipo de mosaico al que figura adosada la inscripción, y especialmente el sistema y tipo de orlas de su parte central parece sugerir una cronología de fines del siglo III y principios del IV d. C., según indicaciones que agradecemos al doctor Abad Casal. (Fig. 5.)

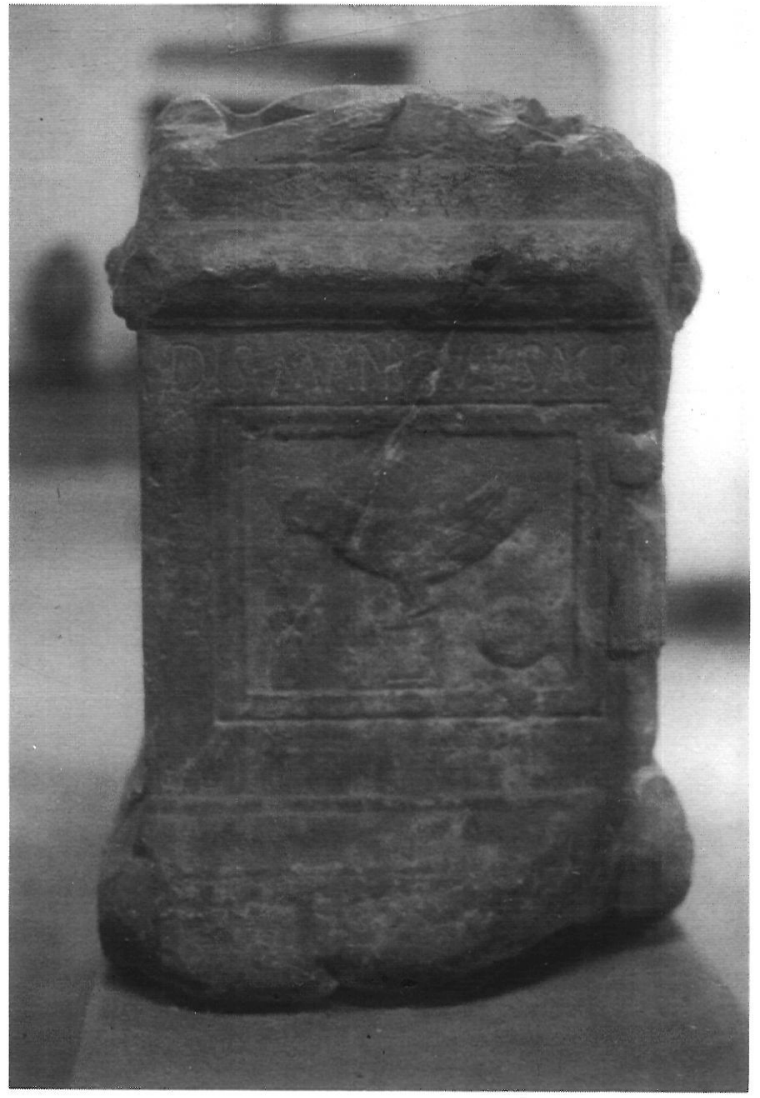

Fig. 6.-Inscripción n. ${ }^{\circ} 36$ (1985, n. $\left.{ }^{\circ} 104\right)$.

Núm. 31.-Rabanal-Abascal 1985, 231, n. ${ }^{\circ} 84$ : Añádase a la bibliografía A. D’Ors, Emerita 28, 1960, 327 ss. = HAE 1974.

Núm. 32.-Rabanal-Abascal 1985, 231, n. ${ }^{\circ} 86$ : Añádase a la bibliografía M. Palomar, Zephyrus 2 , 1951, 21 ss., n. ${ }^{\circ}$ 12. = $\operatorname{HAE} 382$. Se trata de un ladrillo.

Núm. 33.-Rabanal-Abascal 1985, 231, n. ${ }^{\circ} 87$ : Probablemente identificable con EE VIII, 196.

Núm. 34.-Rabanal-Abascal 1985, 232, n. ${ }^{\circ} 92$, fig. 53: Añádase a la bibliografía A. D'Ors, Emerita 28, 1960, 327 ss. = $\operatorname{HAE} 1972$.

Núm. 35.-Rabanal-Abascal 1985, 233-234, n. ${ }^{\circ}$ 96, fig. 56: Añádase a la bibliografía A. D’Ors, Emerita 28, 1960, 327 ss. = HAE 1975.

Núm. 36.-Rabanal-Abascal 1985, 236-237, n. ${ }^{\circ}$ 104, fig. 61: La pieza se encuentra en el Museo de Bellas Artes de Valencia. Sobre su procedencia, 
frente a la hipótesis de Fita, Laborde (1975, planxa CXLIII, n. ${ }^{\circ} 30$ ) supone que se encontró en Villajoyosa. Foto: M. Olcina. Fig. 6.

Núm. 37.—Rabanal-Abascal 1985, 238, n. ${ }^{\circ}$ I: Añádase EE IX, p. 134.

\section{BIBLIOGRAFÍA}

RABANAL, M. A. y ABASCAL, J. M. 1985, «Inscripciones romanas de la provincia de Alicante». Lucentum IV, 191-244.

RAMOS FOLQUÉS, A. 1953, «Carta arqueológica del término municipal de Elche (Alicante)», AEA XXVI, N. ${ }^{\circ} 88,2 .^{\circ}$ semestre, 323ss. 